Pacific Journal of Mathematics

NEW INFINITE CLASSES OF PERIODIC JACOBI-PERRON 


\section{NEW INFINITE CLASSES OF PERIODIC JACOBI-PERRON ALGORITHMS}

\section{LEON BERNSTEIN}

The question whether a system of $n-1$ real algebraic numbers $(n=2,3, \cdots)$ chosen from an algebraic field of degree not higher than $n$, yields periodicity by Jacobi's Algorithm is still as open and challenging as hundred years ago. The present paper gives an affirmative answer to this problem in the following case: let $K(w)$ be an algebraic number field generated by $w=\left(D^{n}-d: m\right)^{1 / n}$, where $m, n, d, D$ are natural numbers satisfying the conditions $m \geqq 1, n \geqq 3, d \mid D, 1 \leqq d \leqq$ $D / 2(n-1)$. Then $n-1$ numbers can be chosen from $K(w)$, so that their Jacobi Algorithm becomes purely periodic. The length of the period equals $n^{2}$ (or $n$, if $d=m=1$ ). This is the longest period of a periodic Jacobi Algorithm ever known. In three corollaries the following special cases are investigated

$$
\begin{array}{ll}
w=\left(D^{n}-d^{r}\right)^{1 / n}, & (\boldsymbol{r}=0,1, \cdots, n) \\
w=\left(D^{n}-d^{r} D\right)^{1 / n}, & (\boldsymbol{r}=0,1, \cdots, n-2) \\
w=\left(D^{n}-p d / m\right)^{1 / n} . & \left(n=p^{u}, p\right. \text { a prime, } \\
& u=1,2, \cdots, m \text { as before })
\end{array}
$$

In all these three cases the Algorithm of Jacobi remains purely periodic with length equal to $n^{2}$.

The main tools in proving these results are the polynomials

$$
\begin{aligned}
f_{s}(w, D-1) & =\sum_{0}^{s}\left(\begin{array}{c}
n-s-1+i \\
i
\end{array}\right) w^{s-i}(D-1)^{i}, \\
F_{s}(w, D) & =\sum_{0}^{s}\left(\begin{array}{c}
n-s-1+i \\
i
\end{array}\right) w^{s-i} D^{i}, \quad(s=1, \cdots, n-1)
\end{aligned}
$$

of which each is an inverse function of the other.

This paper reveals new infinite classes of Periodic Jacobi Algorithms, adding more and wider specific cases to already existing results explored by the author in his previous works. For any given real number $a^{(0)}$ Euclid's Algorithm, namely

$$
a^{(0)}=b^{(0)}+\frac{1}{a^{(1)}}, \cdots, a^{(v)}=b^{(v)}+\frac{1}{a^{(v+1)}}, \quad(v=0,1, \cdots)
$$

where $b^{(v)}=\left[a^{(v)}\right]$ is the greatest integer not exceeding $a^{(v)}$, leads up to Ordinary Continued Fractions. This Algorithm was generalized by Jacobi [1], and its theory masterfully developed by Perron [2] for any

Received June 18, 1964. This paper was supported by the Lord Sieff Foundation. 
number of $n-1$ real numbers ( $\mathrm{n} \geqq 3$ ) in the following way.

Let $a_{k}^{(0)}(k=1,2, \cdots, n-1)$ be any set of $\mathrm{n}-1$ real numbers; from this set (infinitely many) new sets $a_{k}^{(v)}(v=0,1, \cdots ;)$ of $n-1$ real numbers each are being formed by the recursion formula

$$
\begin{aligned}
a_{n-1}^{(v+1)}=\frac{1}{a_{1}^{(v)}-b_{1}^{(v)}} ; \quad a_{k-1}^{(v+1)}=\left(a_{k}^{(v)}-b_{k}^{(v)}\right) a_{n-1}^{(v+1)}, \\
\quad(v=0,1, \cdots ; k=2,3, \cdots, n-1)
\end{aligned}
$$

where again $b_{k}^{(v)}=\left[a_{k}^{(v)}\right]$ is the greatest integer not exceeding $\boldsymbol{a}_{k}^{(v)}$. For $n=2$ Jacobi-Perron's Algorithm (henceforth denoted by JAPAL) is Euclid's Algorithm, namely $a_{1}^{(v+1)}=1:\left(a_{1}^{(v)}-b_{1}^{(v)}\right)$. The JAPAL is called periodic, if there exist two nonnegative integers $t, m$ such that

$$
a_{k}^{(v+m)}=a_{k}^{(v)}, \quad(k=1,2, \cdots, n-1 ; v=t, t+1, \cdots)
$$

whereby the $t$ lines

$$
a_{1}^{(v)}, a_{2}^{(v)}, \cdots, a_{n-1}^{(v)} \quad(v=0,1, \cdots, t-1)
$$

are called the preperiod of the JAPAL, $t$ its length, and the $m$ lines

$$
a_{1}^{(v)}, a_{2}^{(v)}, \cdots, a_{n-1}^{(v)} \quad(v=t, t+1, \cdots, m+t-1)
$$

are called the period of the JAPAL, $m$ its length: the sum $m+t$ is called the length of the JAPAL. For $t=0$ the JAPAL is called purely periodic. Whether or not there exist, for any $n>2$, remarkable classes of sets of $n-1$ real numbers whose JAPAL becomes periodic, could not be decided by Perron.

In eight previous papers [3] I succeeded to prove that the JAPAL becomes periodic for certain sets of $n-1$ Algebraic Irrationals of degree $n$. Some specific results announced in my papers are the following:

Let $D, d, m, n$ be natural numbers such that

$$
n \geqq 3 ; \quad m \geqq 1 ; \quad d \mid D ; \quad D \geqq d C \quad(C \text { a positive constant })
$$

and let $w$ denote one of the following irrationals -

$$
w=\left(D^{n}+d\right)^{1: n} ; \quad\left(D^{n}+d: m\right)^{1: n} ; \quad\left(D^{n}+d^{k} D\right)^{1: n} ; \quad\left(D^{n}-d\right)^{1: n},
$$

then the JAPAL of the $n-1$ numbers

$$
w, w^{2}, \cdots, w^{n-1}
$$

becomes periodic with the lengths $2 n-1 ; 2 n-1 ; 2 n-1 ; n^{2}+(n-1)^{2}$ respectively. Trying to enlarge the family of infinite algebraic fields $K(w)$ containing sets of $n-1$ numbers whose JAPAL becomes periodic, I naturally asked for the periodicity of $\left(D^{n}-d: m\right)^{1: n},\left(D^{n}-d^{k}\right)^{1: n}$ 
$(k=0,1, \cdots, n),\left(D^{n}-d^{k} D\right)^{1: n}(k=0,1, \cdots, n-2)$ and succeeded to establish it. The results are announced in this paper. My previous results thus become a special case of $\left(D^{n}-d: m\right)^{1: n}(m=1)$; but here I use much more refined methods to prove periodicity.

II. Statement of the main theorem. In order to state the main result of this paper it is advisable to introduce the following new notations:

DEFINITION 1. A matrix of $n$ rows and $n-1$ columns of the form

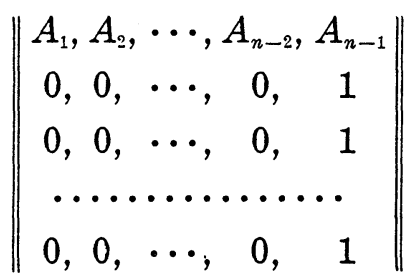

will be called a fugue. The first row of the fugue will be called its accumulator, and the numbers

$$
A_{1}, A_{2}, \cdots, A_{n-1}
$$

the first, second, $\cdots, n-1$ st element of the fugue's accumulator.

DEFINITION 2. The meaning of a combined sigma-sign is given by the formula

$$
\sum_{i=0 ; i c c}^{t-1 / n} a_{i}=c \sum_{i=0}^{t-1} a_{1}+\sum_{i=t}^{n} a_{i}
$$

We are now able to state

The MaIN Theorem. Let $m, n, d, D$ be natural numbers satisfying the following conditions

$$
m \geqq 1 ; n \geqq 3 ; \quad d \mid D ; \quad 1 \leqq d \leqq D: 2(n-1) .
$$

Let us further denote

$$
\begin{aligned}
w & =\left(D^{n}-d: m\right)^{1: n}, \\
f_{s}(w, D-1) & =\sum_{i=0}^{s}\left(\begin{array}{c}
n-1-s+i \\
i
\end{array}\right) w^{s-i}(D-1)^{i}, \quad(s=1, \cdots, n-1,
\end{aligned}
$$

then the JAPAL of the $n-1$ numbers

1 For $n=2$ we get Euclid's Algorithm leading up to the periodic Continued fractions of a quadratic irrational. We shall demonstrate the validity of the Main Theorem in this case, too. 


$$
f_{1}(w, D-1), f_{2}(w, D-1), \cdots, f_{n-1}(w, D-1)
$$

is purely periodic and its primitive length is $n^{2}$. The period consists of $n$ fugues. The $n-1$ elements of the accumulator of the first fugue have the form

$$
A_{k}=-1+\sum_{i=0}^{k}\left(\begin{array}{c}
n-1-k+i \\
i
\end{array}\right) D^{k-i}(D-1)^{i} ;(k=1, \cdots, n-1) .
$$

The accumulator of the sth fugue $(s=2, \cdots, n-1)$ has the form: the first $n-s$ elements have the form

$$
A_{k}=-1+\sum_{i=0}^{k}\left(\begin{array}{c}
n-1-k+i \\
i
\end{array}\right) D^{k-i}(D-1)^{i} ; \quad(k=1, \cdots, n-s)
$$

the following $s-1$ elements have the form

$$
\begin{array}{r}
A_{n-s+t}=-1+\sum_{i=0 / m: d}^{t-1 / n-s+t}(-1)^{i}\left(\begin{array}{c}
s-1-t+i \\
i
\end{array}\right)\left(\begin{array}{c}
n \\
s-t+i
\end{array}\right) D^{n-s+t-i} ; \\
(t=1,2, \cdots, s-1) .
\end{array}
$$

The $n-1$ elements of the accumulator of the $n$th fugue have the form

$$
\begin{array}{r}
A_{n-s+t}=-1+(-1)^{t}\left(\begin{array}{c}
n-1 \\
t
\end{array}\right)+(m: d) \sum_{i=0}^{t-1}(-1)^{i}\left(\begin{array}{c}
n-1-t+i \\
i
\end{array}\right)\left(\begin{array}{c}
n \\
t-i
\end{array}\right) D^{t-i} . \\
(t=1,2, \cdots, n-1)
\end{array}
$$

In the case of $m=d=1$ the primitive length of the period is $n$. The period consists here of one fugue, and the elements of its accumulator have the form (6).

In the quadratic case $(n=2)$ we have, according to the Main Theorem, as can be easily calculated by the reader,

$$
\begin{aligned}
w= & \left(D^{2}-d: m\right)^{1: 2} ; 2 d \leqq D ; d \mid D, \\
& f_{1}(w, D-1)=w+D-1 ;
\end{aligned}
$$

the accumulator of the first fugue has the form

$$
A_{1}=2(D-1) \text {; }
$$

the accumulator of the second fugue has the form

$$
A_{1}=2(m D: d-1) ;
$$

therefore we have the development in a periodic continued fraction:

$$
\left(D^{2}-(d: m)\right)^{1: 2}+D-1=[\overline{2(D-1), 1,2(m D: d-1), 1}] .
$$


NEW INFINITE CLASSES OF PERIODIC JACOBI-PERRON ALGORITHMS 443

Illustration for (6e): $D=12 ; d=3 ; m=10$,

$$
\sqrt{143,7}+11=[\overline{22,1,78,1}] \text {. }
$$

Two conclusions which follow directly from the Main Theorem are the following corollaries:

COROLLARY 1. Let $n, d, D$ be natural numbers satisfying the following conditions:

$$
n \geqq 3 ; \quad d: D ; \quad 1 \leqq d \leqq D: 2(n-1),
$$

and let denote

$$
w=\left(D^{n}-d^{r}\right)^{1: n}, \quad(r=0,1, \cdots, n)
$$

$$
f_{s}(w, D-d)=d^{-s} \sum_{i=0}^{s}\left(\begin{array}{c}
n-1-s+i \\
i
\end{array}\right) w^{s-i}(D-d)^{i}
$$

$$
(s=1, \cdots, n-1)
$$

then the JAPAL of the $n-1$ numbers

$$
f_{1}(w, D-d), f_{2}(w, D-d), \cdots, f_{n-1}(w, D-d)
$$

is purely periodic and its primitive length is $n^{2}$. (the case $d=1$ is excluded). The period consists of $n$ fugues. The $n-1$ elements of the accumulator of the first fugue have the form:

$$
A_{k}=-1+d^{-k} \sum_{i=0}^{k}\left(\begin{array}{c}
n-1-k+i \\
i
\end{array}\right) D^{k-i}(D-d)^{i} ; \quad(k=1, \cdots, n-1)
$$

the elements of the accumulator of the sth fugue $(s=2,3, \cdots, n-1)$ have the form: the first $n-s$ elements have the form-

$$
A_{k}=-1+d^{-k} \sum_{i=0}^{k}\left(\begin{array}{c}
n-1-k+i \\
i
\end{array}\right) D^{k-i}(D-d)^{i} ; \quad(k=1, \cdots, n-s)
$$

the following $s-1$ elements have the form:

$$
\begin{array}{r}
A_{n-s+t}=-1+\sum_{i=0 / d^{n-r}}^{t-1 ; n-s+t}(-1)^{i}\left(\begin{array}{c}
s-1-t+i \\
i
\end{array}\right)\left(\begin{array}{c}
n \\
s-t+i
\end{array}\right)\left(\frac{D}{d}\right)^{n-s+t-i} . \\
(t=1,2, \cdots, s-1)
\end{array}
$$

the $n-1$ elements of the accumulator of the $n$th fugue have-the form:

$$
\begin{aligned}
A_{n-s+t}= & -1+(-1)^{t}\left(\begin{array}{c}
n-1 \\
t
\end{array}\right) \\
& +d^{n-r} \sum_{i=0}^{t-1}(-1)^{i}\left(\begin{array}{c}
n-1-t+i \\
i
\end{array}\right)\left(\begin{array}{c}
n \\
t-i
\end{array}\right)\left(\frac{D}{d}\right)^{n-s+t-\imath} \cdot \\
& (t=1,2, \cdots, r n-1)
\end{aligned}
$$


Corollary 2. Let $n, d, D$ be natural numbers satisfying the following conditions:

$$
n \geqq 3 ; \quad d \mid D ; \quad 2 d(n-1) \leqq D \leqq d^{n-r}, \quad(r=0, \cdots, n-2)
$$

and let denote

$$
w=\left(D^{n}-d^{r} D\right)^{1: n}, \quad(r=0,1, \cdots, n-2)
$$

$$
f_{s}(w, D-d)=d^{-s} \sum_{i=0}^{s}\left(\begin{array}{c}
n-1-s+i \\
i
\end{array}\right) w^{s-i}(D-d)^{i} ;(s=1,2, \cdots, n-1) \text {. }
$$

Then the JAPAL of the $n-1$ numbers

$$
f_{1}(w, D-d), f_{2}(w, D-d), \cdots, f_{n-1}(w, D-1)
$$

is purely periodic and its primitive length is $n^{2}$. The period consists of $n$ fugues. The $n-1$ elements of the accumulator of the first fugue have the form:

(8) $A_{k}=-1+d^{-k} \sum_{i=0}^{k}\left(\begin{array}{c}n-1-k+i \\ i\end{array}\right) D^{k-i}(D-d)^{i} ;(k=1,2, \cdots, n-1)$.

The $n-1$ elements of the accumulator of the sth fugue have the form: $(s=2,3, \cdots, n-1)$ the first $n-s$ elements have the form-

(8a) $A_{k}=-1+d^{-k} \sum_{i=0}^{k}\left(\begin{array}{c}n-1-k+i \\ i\end{array}\right) D^{k-i}(D-d)^{i},(k=1,2, \cdots, n-s)$.

The following $s-1$ elements have the form

$$
\begin{array}{r}
A_{t}=-1+\sum_{i=0 / d^{n-r}: D}^{t-1 / n-s+t}(-1)^{i}\left(\begin{array}{c}
s-t-1+i \\
i
\end{array}\right)\left(\begin{array}{c}
n \\
s-t+i
\end{array}\right)\left(\frac{D}{d}\right)^{n-s+t-i} ; \\
(t=1,2, \cdots, s-1)
\end{array}
$$

The $n-1$ elements of the accumulator of the $n$th fugue have the form:

$$
\begin{aligned}
& A_{t}=-1+\sum_{i=0 / d^{n-r}: D}^{t-1 / n-s+t}(-1)^{i}\left(\begin{array}{c}
s-1-t+i \\
i
\end{array}\right)\left(\begin{array}{c}
n \\
s-t+i
\end{array}\right)\left(\frac{D}{d}\right)^{n-s+t-i} \\
& +(-1)^{t}\left(\begin{array}{c}
n-1 \\
t
\end{array}\right) \text {. } \\
& (t=1,2, \cdots, n-1)
\end{aligned}
$$

It is obvious that all the elements of the accumulators (6) to (8a) are integers. We shall prove that the elements of the accumulators $(8 \mathrm{~b}),(8 \mathrm{c})$ are integers, too. To this end we have to prove that

$$
\left(d^{n-r}: D\right)(D: d)^{n-s-t-i}
$$


are integers. Denoting $n-s+t-i=u$, we have $1 \leqq u \leqq n-1$; further

$$
\left(d^{n-r}: D\right)(D: d)^{u}=D^{u-1}: d^{u-n+r}
$$

Since $d \mid D$, we have to prove $u-n+r \leqq u-1$. But

$$
u-n+r \leqq u-n+n-2=u-2 \text {. }
$$

III. Auxiliary functions-notations and identities. The essential tools used here to prove the Main Theorem and its Corollaries are the following functions:

$$
\begin{gathered}
f_{s}(w, D-1)=\sum_{i=0}^{s}\left(\begin{array}{c}
n-s-1+i \\
i
\end{array}\right) w^{s-i}(D-1)^{i} ; \\
(s=1, \cdots, n-1), f_{0}(w, D-1)=1 . \\
F_{s}(w, D)=\sum_{i=0}^{s}\left(\begin{array}{c}
n-1-s+i \\
i
\end{array}\right) w^{s-i} D^{i} ; \\
(s=1, \cdots, n-1), F_{0}(w, D)=1 . \\
g_{n-s, t}\left(w_{,} D\right)=\sum_{i=0 / m: d}^{t-1 / n-s+t}\left(\begin{array}{c}
-1)^{i}\left(\begin{array}{c}
s-t-1+i \\
i
\end{array}\right) F_{n-s+t-i}(w, D) ; \\
(s=2,3, \cdots, n ; t=1,2, \cdots, s-1)
\end{array}\right.
\end{gathered}
$$

For any polynomial $P_{s}(w, D)$ in $w, D$ with integers $c_{i}$ as coefficients, namely

$$
P_{s}(w, D)=\sum_{i=0}^{s} c_{i} w^{s-i} D^{i} ; \quad(s=1, \cdots, n-1), P_{0}(w, D)=1
$$

the following abbreviations will be used

$$
\begin{array}{lr}
P_{s}(w, D)=P_{s} ; & \left(s=1, \cdots, n-1 ; P_{0}=1\right) . \\
P_{s}(D, D)=\bar{P}_{s} ; & \left(s=1, \cdots, n-1 ; \bar{P}_{0}=1\right) . \\
\frac{P_{s}(D, D)-P_{s}(w, D)}{P_{1}(D, D)-P_{1}(w, D)}=\frac{\bar{P}_{s}-P_{s}}{\bar{P}_{1}-P_{1}}={ }^{(1)} P_{s} ; \\
\left(s=1, \cdots, n-1 ;{ }^{(1)} P_{0}=0\right) .
\end{array}
$$

The following identities are essential for the proof of the Main Theorem and its corollaries:

$$
f_{s}(D-1, D-1)=\left(\begin{array}{l}
n \\
s
\end{array}\right)(D-1)^{s} ; \quad(s-0,1, \cdots, n-1) .
$$

Proof of (13). We have from (9): 


$$
\begin{aligned}
& f_{s}(D-1, D-1)=\sum_{i=0}^{s}\left(\begin{array}{c}
n-1-s+i \\
i
\end{array}\right)(D-1)^{s-i}(D-1)^{i} \\
&=(D-1)^{s} \sum_{i=0}^{s}\left(\begin{array}{c}
n-1-s+i \\
i
\end{array}\right)=(D-1)^{s}\left(\begin{array}{c}
n \\
s
\end{array}\right) . \\
& \text { a) } \quad(s=0,1, \cdots, n-1)
\end{aligned}
$$

Proof of (13a). This is completely analogous to proof of (13).

$$
{ }^{(1)} F_{s}=F_{s-1} ; \quad(s=1,2, \cdots, n-1) \text {. }
$$

Proof of (14). We have from (10)-

$$
\begin{aligned}
F_{1} & =\sum_{i=0}^{1}\left(\begin{array}{c}
n-2+i \\
i
\end{array}\right) w^{1-i} D^{i}=v+(n-1) D ; \\
\bar{F}_{1} & =D+(n-1) D=n D ; \\
F_{1}-\bar{F}_{1} & =w-D .
\end{aligned}
$$

We thus have to prove

$$
F_{s}-\bar{F}_{s}=(w-D) F_{s-1}
$$

We have

$$
\begin{aligned}
& F_{s}-\bar{F}_{s}=\sum_{i=0}^{s}\left(\begin{array}{c}
n-1-s+i \\
i
\end{array}\right) w^{s-i} D^{i}-\left(\begin{array}{l}
n \\
s
\end{array}\right) D^{s} \\
& (w-D) F_{s-1}=(w-D) \sum_{i=0}^{s-1}\left(\begin{array}{c}
n-s+i \\
i
\end{array}\right) w^{s-1-i} D^{i} \\
& =\sum_{i=0}^{s-1}\left(\begin{array}{c}
n-s+i \\
i
\end{array}\right) w^{s-i} D^{i}-\sum_{i=0}^{s-1}\left(\begin{array}{c}
n-s+i \\
i
\end{array}\right) w^{s-i-1} D^{i+1} \\
& =\sum_{i=0}^{s-1}\left(\begin{array}{c}
n-s+i \\
i
\end{array}\right) w^{s-i} D^{i}-\sum_{i=1}^{s}\left(\begin{array}{c}
n-s+i-1 \\
i-1
\end{array}\right) w^{s-i} D^{i} \\
& =w^{s}+\sum_{i=1}^{s-1}\left(\left(\begin{array}{c}
n-s+i \\
i
\end{array}\right)-\left(\begin{array}{c}
n-s+i-1 \\
i-1
\end{array}\right)\right) w^{s-i} D^{i}-\left(\begin{array}{c}
n-1 \\
s-1
\end{array}\right) D^{s} \\
& =w^{s}+\sum_{i=1}^{s-1}\left(\begin{array}{c}
n-s-1+i \\
i
\end{array}\right) w^{s-i} D^{i}+\left(\begin{array}{c}
n-1 \\
s
\end{array}\right) D^{s}-\left(\begin{array}{l}
n \\
s
\end{array}\right) D^{s} \\
& =\sum_{i=0}^{s}\left(\begin{array}{c}
n-s-1+i \\
i
\end{array}\right) w^{s-i} D^{i}-\left(\begin{array}{l}
n \\
s
\end{array}\right) D^{s} \text {. } \\
& f_{s}=\sum_{i=0}^{s}(-1)^{i}\left(\begin{array}{c}
n-1-s+i \\
i
\end{array}\right) F_{s-i} ; \quad(s=0,1, \cdots, n-1) .
\end{aligned}
$$


Proof of (15). If we arrange the expression on the right hand of the equation

$$
\begin{aligned}
f_{s} & =\sum_{i=0}^{s}(-1)^{i}\left(\begin{array}{c}
n-1-s+i \\
i
\end{array}\right) F_{s-i} \\
& =\sum_{i=0}^{s}(-1)^{i}\left(\begin{array}{c}
n-1-s+i \\
i
\end{array}\right) \sum_{j=0}^{s-i}\left(\begin{array}{c}
n-1-s+i+j \\
j
\end{array}\right) w^{s-i-j} D^{j}
\end{aligned}
$$

in descending powers of $w$, we get

$$
f_{s}=\sum_{i=0}^{s} w^{s-i} \sum_{j=0}^{i}(-1)^{s}\left(\begin{array}{c}
n-s-1+j \\
j
\end{array}\right)\left(\begin{array}{c}
n-s-1+i \\
i-j
\end{array}\right) D^{i-j}
$$

Now the identity holds:

$$
\begin{gathered}
\left(\begin{array}{c}
n-s-1+j \\
j
\end{array}\right)\left(\begin{array}{c}
n-s-1+i \\
i-j
\end{array}\right)=\frac{(n-s-1+j) !(n-s-1+i) !}{(n-s-1) ! j !(i-j) !(n-s-1+j) !} \\
=\frac{(n-s-1+i) !}{j !(n-s-1) !(i-j) !}=\frac{(n-s-1+i) ! i !}{i !(n-s-1) ! j !(i-j) !} \\
=\left(\begin{array}{c}
n-s-1+i \\
i
\end{array}\right)\left(\begin{array}{l}
i \\
j
\end{array}\right) .
\end{gathered}
$$

In view of this identity we get

$$
\begin{aligned}
f_{s} & =\sum_{i=0}^{s} w^{s-1}\left(\begin{array}{c}
n-s-1+i \\
i
\end{array}\right) \sum_{j=0}^{i}(-1)^{j}\left(\begin{array}{l}
i \\
j
\end{array}\right) D^{i-j} \\
& =\sum_{i=0}^{s}\left(\begin{array}{c}
n-s-1+i \\
i
\end{array}\right) w^{s-i}(D-1)^{i} .
\end{aligned}
$$

$$
{ }^{(1)} f_{s}=\sum_{i=0}^{s-1}(-1)^{i}\left(\begin{array}{c}
n-1-s+i \\
i
\end{array}\right) F_{s-1-i} . \quad(s=1,2, \cdots, n-1) \text {. }
$$

Proof of (16). We have from (15):

$$
\begin{aligned}
& f_{s}=\sum_{i=0}^{s-1}(-1)^{i}\left(\begin{array}{c}
n-1-s+i \\
i
\end{array}\right) F_{s-i}+(-1)^{s}\left(\begin{array}{c}
n-1 \\
s
\end{array}\right), \\
& \bar{f}_{s}=\sum_{i=0}^{s-1}(-1)^{i}\left(\begin{array}{c}
n-1-s+i \\
i
\end{array}\right) \bar{F}_{s-i}+(-1)^{s}\left(\begin{array}{c}
n-1 \\
s
\end{array}\right) .
\end{aligned}
$$

In view of these two formulas and according to (14), we get

$$
\begin{aligned}
& { }^{(1)} f_{s}=\frac{\bar{f}_{s}-f_{s}}{D-w}=\sum_{i=0}^{s-1}(-1)^{i}\left(\begin{array}{c}
n-1-s+i \\
i
\end{array}\right)\left(\bar{F}_{s-i}-F_{s-i}\right):(D-w) \\
& =\sum_{i=0}^{s-1}(-1)^{i}\left(\begin{array}{c}
n-1-s+i \\
i
\end{array}\right) F_{s-1-i} \text {. }
\end{aligned}
$$


Proof of (17). We have, on the basis of (16)

$$
\begin{aligned}
& \text { (1) } f_{8}={ }^{(1)} f_{8-1} \\
& =\sum_{i=0}^{s-1}(-1)^{i}\left(\begin{array}{c}
n-1-s+i \\
i
\end{array}\right) F_{s-1-i} \\
& -\sum_{i=0}^{s-2}(-1)^{i}\left(\begin{array}{c}
n-s+i \\
i
\end{array}\right) F_{s-2-i} \\
& =F_{s-1}+\sum_{i=1}^{s-1}(-1)^{i}\left(\begin{array}{c}
n-1-s+i \\
i
\end{array}\right) F_{s-1-i} \\
& -\sum_{i=0}^{s-2}(-1)^{i}\left(\begin{array}{c}
n-s+i \\
i
\end{array}\right) F_{s-2-i} \\
& =F_{s-1}-\sum_{i=0}^{s-2}(-1)^{i}\left(\begin{array}{c}
n-s+i \\
i+1
\end{array}\right) F_{s-2-i} \\
& -\sum_{i=0}^{s-2}(-1)^{i}\left(\begin{array}{c}
n-s+i \\
i
\end{array}\right) F_{s-2-i} \\
& =F_{s-1}-\sum_{i=0}^{s-2}(-1)^{i}\left(\left(\begin{array}{c}
n-s+i \\
i+1
\end{array}\right)+\left(\begin{array}{c}
n-s+i \\
i
\end{array}\right)\right) F_{s-2-i} \\
& =F_{s-1}-\sum_{i=0}^{s-2}(-1)^{i}\left(\begin{array}{c}
n-s+i+1 \\
i+1
\end{array}\right) F_{s-2-i} \\
& =F_{s-1}+\sum_{i=1}^{s-1}(-1)^{i}\left(\begin{array}{c}
n-s+i \\
i
\end{array}\right) F_{s-1-i} \\
& =\sum_{i=0}^{s-1}(-1)^{i}\left(\begin{array}{c}
n-s+i \\
i
\end{array}\right) F_{s-1-i}=f_{s-1} \text {. } \\
& { }^{(1)} g_{n-8, t}=\sum_{i=0 / m: d}^{t-1 / n-s+t-1}(-1)^{i}\left(\begin{array}{c}
s-t-1+i \\
i
\end{array}\right) F_{n-8+t-1-i} \\
& (s=2,3, \cdots, n ; t=1,2, \cdots, s-1) \text {. }
\end{aligned}
$$

Proof of (18). This follows directly from (16), if we interprete ${ }^{(1)} g_{n-8, t}$ as $\left(\bar{g}_{n-8, t}-g_{n-t, 8}\right):(D-w)$. (It will be shown later that this interpretation is in accordance with the general notation of ${ }^{(1)} P_{s}$.

$$
{ }^{(1)} g_{n-8,1}-{ }^{(1)} f_{n-8}=g_{n-(8+1), 1} . \quad(s=1,2, \cdots, n-1) .
$$

Proof of (19). We have from (16), (18):

$$
\begin{aligned}
& { }^{(1)} g_{n-s, 1}-{ }^{(1)} f_{n-s} \\
& \quad=\frac{m}{d} F_{n-s}+\sum_{i=1}^{n-s}(-1)^{i}\left(\begin{array}{c}
s-2+i \\
i
\end{array}\right) F_{n-8-i}
\end{aligned}
$$




$$
\begin{aligned}
&-\sum_{i=0}^{n-s-1}(-1)^{i}\left(\begin{array}{c}
s-1+i \\
i
\end{array}\right) F_{n-s-1-i} \\
&= \frac{m}{d} F_{n-s}+\sum_{i=0}^{n-s-1}(-1)^{i+1}\left(\begin{array}{c}
s-1+i \\
i+1
\end{array}\right) F_{n-s-1-i} \\
&-\sum_{i=0}^{n-s-1}(-1)^{i}\left(\begin{array}{c}
s-1+i \\
i
\end{array}\right) F_{n-s-1-i} \\
&= \frac{m}{d} F_{n-s}+\sum_{i=0}^{n-s-1}(-1)^{i+1}\left(\left(\begin{array}{c}
s-1+i \\
i+1
\end{array}\right)+\left(\begin{array}{c}
s-1+i \\
i
\end{array}\right)\right) F_{n-s-1-i} \\
&= \frac{m}{d} F_{n-s}+\sum_{i=0}^{n-s-1}(-1)^{i+1}\left(\begin{array}{c}
s+i \\
i+1
\end{array}\right) F_{n-8-1-i} \\
&= \frac{m}{d} F_{n-s}+\sum_{i=1}^{n-s}(-1)^{i}\left(\begin{array}{c}
s-1+i \\
i
\end{array}\right) F_{n-s-i} \\
&={ }_{i=0 / m: d}(-1)^{i}\left(\begin{array}{c}
s-1+i \\
i
\end{array}\right) F_{n-8-i}=g_{n-(s+1), 1} . \\
&{ }^{(1)} g_{n-8, t+1}-{ }^{(1)} g_{n-s, t}=g_{n-(s+1), t+1} \\
&(s=2, \cdots, n-1 ; t=1, \cdots, s-1) .
\end{aligned}
$$

Proof of (20). We have from (18):

$$
\begin{aligned}
& \text { (1) } g_{n-s, t+1}-{ }^{(1)} g_{n-s, t} \\
& =\sum_{i=0 / m: d}^{t / n-s+t}(-1)^{i}\left(\begin{array}{c}
s-t-2+i \\
i
\end{array}\right) F_{n-s+t-i} \\
& -\sum_{i=0 / m: d}^{t-1 / n-s+t-1}(-1)^{i}\left(\begin{array}{c}
s-t-1+i \\
i
\end{array}\right) F_{n-s+t-1-i} \\
& =\frac{m}{d} \sum_{i=0}^{t}(-1)^{i}\left(\begin{array}{c}
s-t-2+i \\
i
\end{array}\right) F_{n-s+t-i} \\
& -\frac{m}{d} \sum_{i=0}^{t-1}(-1)^{i}\left(\begin{array}{c}
s-t-1+i \\
i
\end{array}\right) F_{n-s+t-1-i} \\
& +\sum_{i=t+1}^{n-s+t}(-1)^{i}\left(\begin{array}{c}
s-t-2+i \\
i
\end{array}\right) F_{n-s+t-i} \\
& -\sum_{i=t}^{n-s+t-1}(-1)^{i}\left(\begin{array}{c}
s-t-1+i \\
i
\end{array}\right) F_{n-s+t-1-i} \\
& =\frac{m}{d} \sum_{i=0}^{t}(-1)^{i}\left(\begin{array}{c}
s-t-2+i \\
i
\end{array}\right) F_{n-s+t-i} \\
& -\frac{m}{d} \sum_{i=1}^{t}(-1)^{i-1}\left(\begin{array}{c}
s-t-2+i \\
i-1
\end{array}\right) F_{n-s+t-i} \\
& +\sum_{i=t+1}^{n-s+t}(-1)^{i}\left(\begin{array}{c}
s-t-2+i \\
i
\end{array}\right) F_{n-s+t-i}
\end{aligned}
$$




$$
\begin{aligned}
& -\sum_{i=t+1}^{n-s+t}(-1)^{i-1}\left(\begin{array}{c}
s-t-2+i \\
i-1
\end{array}\right) F_{n-8+t-i} \\
= & \frac{m}{d} F_{n-s+t}+\frac{m}{d} \sum_{i=1}^{t}(-1)^{i}\left(\begin{array}{c}
s-t-1+i \\
i
\end{array}\right) F_{n-s+t-i} \\
& +\sum_{i=t+1}^{n-s+t}(-1)^{i}\left(\begin{array}{c}
s-t-1+i \\
i
\end{array}\right) F_{n-s+t-i} \\
= & \sum_{i=0 / m: d}^{t / n-8+t}(-1)^{i}\left(\begin{array}{c}
s-t-1+i \\
i
\end{array}\right) F_{n-s+t-i}=g_{n-(s+1), t+1} .
\end{aligned}
$$

(20a)

$$
{ }^{(1)} g_{n-8, t}-{ }^{(1)} g_{n-8, q}=\sum_{i=0}^{t-1-q} g_{n-(8+1), t-i} \text {. }
$$

Proof of (20a). We have from (20)

$$
\text { (1) } \begin{aligned}
g_{n-8, t}-{ }^{(1)} g_{n-8, q} & =\sum_{i=0}^{t-1-q}\left({ }^{(1)} g_{n-8, t-i}-{ }^{(1)} g_{n-8, t-i-1}\right) \\
& =\sum_{i=0}^{t-1-q}{ }^{(1)} g_{n-(8+1), t-i}
\end{aligned}
$$

(20b)

$$
{ }^{(1)} g_{0, t}-{ }^{(1)} g_{0, t-1}=\frac{m}{d} f_{t-1} . \quad(t=1,2, \cdots, n-1) \text {. }
$$

Proof of (20b). From (18) we derive:

$$
\begin{aligned}
{ }^{(1)} g_{0, t} & =\frac{m}{d} \sum_{i=0}^{t-1}(-1)^{i}\left(\begin{array}{c}
n-t-1+i \\
i
\end{array}\right) F_{t-1-i}, \\
{ }^{(1)} g_{0, t-1} & =\frac{m}{d} \sum_{i=0}^{t-2}(-1)^{i}\left(\begin{array}{c}
n-t+i \\
i
\end{array}\right) F_{t-2-i} ;
\end{aligned}
$$

$$
\begin{aligned}
{ }^{(1)} g_{0, t}-{ }^{(1)} g_{0, t-1} & \\
= & \frac{m}{d} \sum_{i=0}^{t-1}(-1)^{i}\left(\begin{array}{c}
n-t-1+i \\
i
\end{array}\right) F_{t-1-i} \\
& -\frac{m}{d} \sum_{i=0}^{t-2}(-1)^{i}\left(\begin{array}{c}
n-t+i \\
i
\end{array}\right) F_{t-2-i} \\
= & \frac{m}{d} F_{t-1}+\frac{m}{d} \sum_{i=1}^{t-1}(-1)^{i}\left(\begin{array}{c}
n-t-1+i \\
i
\end{array}\right) F_{t-1-i} \\
& +\frac{m}{d} \sum_{i=1}^{t-1}(-1)^{i}\left(\begin{array}{c}
n-t-1+i \\
i-1
\end{array}\right) F_{t-1-i} \\
= & \frac{m}{d} F_{t-1}+\frac{m}{d} \sum_{i=1}^{t-1}(-1)^{i}\left(\begin{array}{c}
n-t+i \\
i
\end{array}\right) F_{t-1-i} \\
= & \frac{m}{d} \sum_{i=0}^{t-1}(-1)^{i}\left(\begin{array}{c}
n-t+i \\
i
\end{array}\right) F_{t-1-i}=\frac{m}{d} f_{t-1} .
\end{aligned}
$$


(20c)

$$
\frac{g_{n-s, s-1}-\bar{g}_{n-s, s-1}+1}{D-w}=g_{n-(s+1), s} . \quad(s=2,3, \cdots, n-1) .
$$

Proof of (20c). We have from (18) and the definition of $w$

$$
\begin{aligned}
\frac{g_{n-8, s-1}-\bar{g}_{n-s, s-1}+1}{D-w} & =\frac{1}{D-w}-{ }^{(1)} g_{n-s, s-1} \\
& =\frac{w^{n-1}+w^{n-2} D+\cdots+D^{n-1}}{D^{n}-w^{n}}-{ }^{(1)} g_{n-8, s-1} \\
& =\frac{F_{n-1}}{D^{n}-\left(D^{n}-d: m\right)}-{ }^{(1)} g_{n-8, s-1} \\
& =\frac{m}{d} F_{n-1}-{ }^{(1)} g_{n-s, s-1} \\
& =\frac{m}{d} F_{n-1}-\sum_{i=0 / m: d}^{s-2 / n-2}(-1)^{2} F_{n-2-i} \\
& =\frac{m}{d} F_{n-1}+\sum_{i=1 / m: d}^{s-1 / n-1}(-1)^{i} F_{n-1-i} \\
& =\sum_{i=0 / m: d}^{s-1 / n-1}(-1)^{i} F_{n-1-i}=g_{n-(s+1), s} .
\end{aligned}
$$

$(20 \mathrm{~d})$

$$
\frac{g_{0, n-1}-\bar{g}_{0, n-1}+1}{(m: d)(D-w)}=f_{n-1} \text {. }
$$

Proof of (20d). We have from previous proofs and formulas

$$
\begin{aligned}
\frac{1}{(m: d)(D-w)} & =F_{n-1} ; \frac{\bar{g}_{0, n-1}-g_{0, n-1}}{(m: d)(D-w)}=\frac{(1) g_{n-n, n-1}}{m: d} \\
& =\frac{d}{m} \sum_{i=0 / m: d}^{n-2}(-1)^{i} F_{n-2-i}=\sum_{i=0}^{n-2}(-1)^{i} F_{n-2-i}
\end{aligned}
$$

therefore

$$
\begin{aligned}
\frac{g_{0, n-1}-\bar{g}_{0, n-1}+1}{(m: d)(D-w)} & =F_{n-1}-\sum_{i=0}^{n-2}(-1)^{i} F_{n-2-i} \\
& =\sum_{i=0}^{n-1}(-1)^{i} F_{n-1-i}=f_{n-1} . \\
{ }^{(1)} f_{s}-{ }^{(1)} f_{q}=\sum_{i=0}^{s-q-1} f_{s-1-i} . & (1 \leqq q \leqq s-1) .
\end{aligned}
$$

Proof of (17a). We have from (17)

$$
{ }^{(1)} f_{s}-{ }^{(1)} f_{q}=\sum_{i=0}^{s-q-1}\left({ }^{(1)} f_{s-i}-{ }^{(1)} f_{s-i-1}\right)=\sum_{i=0}^{s-q-1} f_{s-1-i} .
$$

(20e) ${ }^{(1)} g_{n-s, t}-{ }^{(1)} f_{q}=\sum_{i=0}^{t-1} g_{n-(s+1) t-i}+\sum_{i=0}^{n-s-q-1} f_{n-s-1-i} . \quad(1 \leqq q \leqq n-s)$.

Proof of (20e). This follows immediately combining (17a), (19), (20a). 
IV. Inequalities. In this chapter we shall establish magnitude relations between the auxiliary functions $f_{s}, F_{s}, g_{n-8, t}$. We first note that

$$
D-1<w<D ; \quad(D-1)^{k}<w^{k}<D^{k} . \quad(k=1,2, \cdots, n-1)
$$

(21) follows directly from the definition of $w$. From (21) and the definition of $f_{\mathrm{s}}$ and $F_{\mathrm{s}}$ follows further

$$
\begin{gathered}
f_{s}(D-1, D-1)<f_{s}<F_{s}<\bar{F}_{s} ; \quad f_{s}<\bar{f}_{s}<\bar{F}_{s} . \\
(s=1,2, \cdots, n-1) \\
\left(1+\frac{1}{D-1}\right)^{n-2}<1,65 \text { for } 2(n-1) \leqq D .
\end{gathered}
$$

Proof of (23). Since $D \geqq 2 d(n-1), d \geqq 1$, we have $D \geqq 2(n-1)$, $D-1 \geqq 2(n-1)-1>2(n-2)$. Therefore

$$
\begin{gathered}
\begin{array}{c}
\left(1+\frac{1}{D-1}\right)^{n-2}<\left(1+\frac{1}{2(n-2)}\right)^{n-2}=\left(\left(1+\frac{1}{2(n-2)}\right)^{2(n-2)}\right)^{1: 2} \\
<e^{1: 2}=1,64872 \cdots<1,65 . \\
\bar{F}_{i} \leqq F_{i+1}(D-1, D-1) .
\end{array} \quad(i=0,1, \cdots, n-2) .
\end{gathered}
$$

Proof of (24). We have to prove, following (13a):

$$
\begin{aligned}
\left(\begin{array}{c}
n \\
i
\end{array}\right) D^{i} & \leqq\left(\begin{array}{c}
n \\
i+1
\end{array}\right)(D-1)^{i+1}, \\
\left(1+\frac{1}{D-1}\right)^{i} & \leqq \frac{n-i}{i+1}(D-1), \\
D-1 & \geqq \frac{i+1}{n-i}\left(1+\frac{1}{D-1}\right)^{i} .
\end{aligned}
$$

We prove a fortiori, since $(i+1):(n-i)$ is an increasing function,

$$
D-1 \geqq \frac{n-1}{2} \cdot 1,65>\frac{i+1}{n-i}\left(1+\frac{1}{D-1}\right)^{i} ;
$$

but

(24a)

$$
D-1 \geqq 2 d(n-1)-1 \geqq 2(n-1)-1>1,65 \frac{n-1}{2} .
$$

$$
\begin{aligned}
F_{s} & <F_{s+t} \cdot \\
& (s=0, \cdots, n-2 ; t=1, \cdots, n-1 ; 1 \leqq s+t \leqq n-1)
\end{aligned}
$$

Proof of (24a). It follows from (22), (24)

$$
F_{s}<\bar{F}_{s}<F_{s+1}(D-1, D-1)<F_{s+1},
$$


NEW INFINITE CLASSES OF PERIODIC JACOBI-PERRON ALGORITHMS 453

so that

$$
F_{s}<F_{s+1}<F_{s+2}<\cdots<F_{s+t}
$$

$$
f_{s}<f_{s+t}
$$

$$
(s=0, \cdots, n-2 ; t=1, \cdots, n-1 ; 1 \leqq s+t \leqq n-1) .
$$

Proof of (24b). It follows from (22), (24)

$$
f_{s}<\bar{f}_{s}<\bar{F}_{s}<F_{s+1}(D-1, D-1)=f_{s+1}(D-1, D-1)<f_{s+1} \text {. }
$$

$$
2 F_{n-2}<\frac{1}{d} F_{n-1}
$$

Proof of (25). We have to prove

$$
2 \sum_{i=0}^{n-2}(i+1) w^{n-2-i} D^{i}<\frac{1}{d} \sum_{i=0}^{n-1} w^{n-1-i} D^{i}
$$

and prove a fortiori

$$
\begin{aligned}
2 \sum_{i=0}^{n-2}(i+1) w^{n-2-i} D^{i} & <\frac{2(n-1)}{D} \sum_{i=0}^{n-1} w^{n-1-i} D^{i} \\
& \leqq \frac{1}{d} \sum_{i=0}^{n-1} w^{n-1-i} D^{i} .
\end{aligned}
$$

We thus have to prove

$$
\sum_{i=0}^{n-2}(i+1) w^{n-2-i} D^{i}<\frac{(n-1) w^{n-1}}{D}+\sum_{i=0}^{n-2}(n-1) w^{n-2-i} D^{i}
$$

which is always true, since $i+1 \leqq n-1,0<(n-1) w^{n-1} / D$,

$$
{ }^{(1)} f_{s} \leqq F_{s-1} . \quad(s=1,2, \cdots, n-1) .
$$

Proof of (26). We have from (15)

${ }^{(1)} f_{s}=F_{s-1}$

$$
\begin{aligned}
& -\sum_{i=1}^{(s-1) / 2}\left(\left(\begin{array}{c}
n-s+2 i-2 \\
2 i-1
\end{array}\right) F_{s-2 i}-\left(\begin{array}{c}
n-s+2 i-1 \\
2 i
\end{array}\right) F_{s-2 i-1}\right) \\
& -e\left(\begin{array}{c}
n-2 \\
s-1
\end{array}\right), \quad(e=0, \text { when } s \text { is odd, }=1 \text { otherwise })
\end{aligned}
$$

so that ${ }^{(1)} f_{s} \leqq F_{8-1}$, if we can prove that the expression under the sigma sign is not negative. We shall therefore prove

$$
\left(\begin{array}{c}
n-s+2 i-2 \\
2 i-1
\end{array}\right) F_{s-2 i}>\left(\begin{array}{c}
n-s+2 i-1 \\
2 i
\end{array}\right) F_{s-2 i-1},
$$

or 


$$
F_{s-2 i}>\frac{n-s+2 i-1}{2 i} F_{s-2 i-1},
$$

and prove a fortiori

$$
\begin{aligned}
F_{s-2 i} & >\left(\begin{array}{c}
n \\
s-2 i
\end{array}\right)(D-1)^{s-2 i} \geqq \frac{n-s+2 i-1}{2 i}\left(\begin{array}{c}
n \\
s-2 i-1
\end{array}\right) D^{s-2 i-1} \\
& >\frac{n-s+2 i-1}{2 i} F_{s-2 i-1} .
\end{aligned}
$$

We thus have to prove

$$
\left(\begin{array}{c}
n \\
s-2 i
\end{array}\right)(D-1)^{s-2 i} \geqq \frac{n-s+2 i-1}{2 i} D^{s-2 i-1},
$$

or

$$
(D-1)^{s-2 i} \geqq \frac{(n-s+2 i-1)(s-2 i)}{2 i(n-s+2 i+1)} D^{s-2 i-1},
$$

or

$$
(D-1) \geqq \frac{n-s+2 i-1}{n-s+2 i+1} \cdot \frac{s-2 i}{2 i}\left(1+\frac{1}{D-1}\right)^{s-2 i-1} .
$$

But from $D \geqq 2 d(n-1)$ we have

$$
D-1>\frac{n-3}{2} \cdot 1,65>\frac{n-s+2 i-1}{n-s+2 i+1} \cdot \frac{s-2 i}{2 i}\left(1+\frac{1}{D-1}\right)^{s-2 i-1} \text {. }
$$

$$
{ }^{(1)} g_{n-8, t}<(m: d) F_{n-8+t-1} . \quad(s=2,3, \cdots, n ; t=1,2, \cdots, s-1) \text {. }
$$

Proof of (27). We have from (18) for $t=2 r+1$

$$
\begin{aligned}
& { }^{(1)} g_{n-s, t}=(m: d) F_{n-s+t-1} \\
& -(m: d) \sum_{i=1}^{r}\left(\left(\begin{array}{c}
s-t+2 i-2 \\
2 i-1
\end{array}\right) F_{n-s+t-2 i}-\left(\begin{array}{c}
s-t+2 i-1 \\
2 i
\end{array}\right) F_{n-s+t-2 i-1}\right) \\
& -\sum_{i=2 r+1}^{n-s+2 r}\left(\left(\begin{array}{c}
s-t+2 i-2 \\
2 i-1
\end{array}\right) F_{n-s+t-2 i}-\left(\begin{array}{c}
s-t+2 i-1 \\
2 i
\end{array}\right) F_{n-s+t-2 i-1}\right) .
\end{aligned}
$$

We shall now prove that the expressions under both the sigma signs are nonnegative, so that ${ }^{(1)} g_{n-8, t}<(m: d) F_{n-8+t-1}$. We have to prove

$$
\left(\begin{array}{c}
s-t+2 i-2 \\
2 i-1
\end{array}\right) F_{n-8+t-2 i}>\left(\begin{array}{c}
s-t+2 i-1 \\
2 i
\end{array}\right) F_{n-8+t-2 i-1},
$$

or

$$
F_{n-s+t-2 i}>\frac{s-t+2 i-1}{2 i} F_{n-s+t-2 i-1} .
$$


We shall prove a fortiori

$$
\begin{aligned}
F_{n-s+t-2 i} & >\left(\begin{array}{c}
n \\
s-t+2 i
\end{array}\right)(D-1)^{n-s+t-2 i} \\
& \geqq \frac{s-t+2 i-1}{2 i}\left(\begin{array}{c}
n \\
s-t+2 i+1
\end{array}\right) D^{n-s+t-2 i-1} \\
& >\frac{s-t+2 i-1}{2 i} F_{n-s+t-2 i-1}
\end{aligned}
$$

We have to prove

$\left(\begin{array}{c}n \\ s-t+2 i\end{array}\right)(D-1)^{n-s+t-2 i} \geqq \frac{s-t+2 i-1}{2 i}\left(\begin{array}{c}n \\ s-t+2 i+1\end{array}\right) D^{n-s+t-2 i-1}$,

or

$$
D-1 \geqq \frac{s-t+2 i-1}{s-t+2 i+1} \cdot \frac{n-s+t-2 i}{2 i} \cdot\left(1+\frac{1}{D-1}\right)^{n-s+t-2 i-1},
$$

which follows immediately from $2(n-1)-1 \leqq D-1$ and the upper and lower bounds of $s, t$, as at the end of the previous proof.

For $t=2 r+2$ we have

$$
\begin{aligned}
& { }^{(1)} g_{n-8, t}=(m: d) F_{n-s+t-1} \\
& -(m: d) \sum_{i=1}^{r}\left(\left(\begin{array}{c}
s-t+2 i-2 \\
2 i-1
\end{array}\right) F_{n-s+t-2 i}-\left(\begin{array}{c}
s-t+2 i-1 \\
2 i
\end{array}\right) F_{n-s+t-2 i-1}\right) \\
& -\left((m: d)\left(\begin{array}{c}
s-2 \\
t-1
\end{array}\right) F_{n-s}-\left(\begin{array}{c}
s-1 \\
t
\end{array}\right) F_{n-s-1}\right) \\
& -\sum_{i=2 r+3}^{n-s+2 r+1}\left(\left(\begin{array}{c}
s-t+2 i-2 \\
2 i-1
\end{array}\right) F_{n-s+t-2 i}-\left(\begin{array}{c}
s-t+2 i-1 \\
2 i
\end{array}\right) F_{n-s+t-2 i-1}\right),
\end{aligned}
$$

so that in order to prove (27) in this case of $t=2 r+2$ we have only to add the proof of

$$
(m: d)\left(\begin{array}{c}
s-2 \\
t-1
\end{array}\right) F_{n-s}-\left(\begin{array}{c}
s-1 \\
t
\end{array}\right) F_{n-s-1} \geqq 0 .
$$

Since $m \geqq 1$, we prove $a$ fortiori

$$
\frac{1}{d}\left(\begin{array}{l}
s-2 \\
t-1
\end{array}\right) F_{n-s} \geqq\left(\begin{array}{c}
s-1 \\
t
\end{array}\right) F_{n-s-1},
$$

or

$$
F_{n-s} \geqq \frac{(s-1) d}{t} F_{n-s-1} .
$$


We prove a fortiori

$$
F_{n-s}>\left(\begin{array}{c}
n \\
s
\end{array}\right)(D-1)^{n-s} \geqq \frac{(s-1) d}{t}\left(\begin{array}{c}
n \\
s+1
\end{array}\right) D^{n-s-1},
$$

or

$$
D-1 \geqq \frac{s-1}{s+1} \cdot \frac{n-s}{t} \cdot d\left(\frac{1}{D-1}\right)^{n-s-1},
$$

which follows immediately from $D-1 \geqq 2 d(n-1)-1>d(n-2) \cdot 1,65$.

$$
g_{n-8, t}<(m: d) F_{n-s+t} . \quad(s=2,3, \cdots, n ; t=1,2, \cdots, s-1) .
$$

Proof of (28). This is completely analogous to proof of (27).

$$
\left[f_{s}\right]=-1+\bar{f}_{s} . \quad(s=1,2, \cdots, n-1) .
$$

Proof of (29). We have to prove

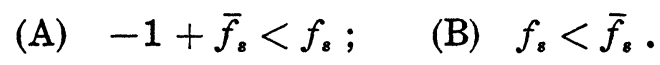

To prove (A) we have to show that

$$
\begin{aligned}
\bar{f}_{s}-f_{s} & <1, \quad \text { or, dividing by } D-w>0, \\
{ }^{(1)} f_{s} & <(m: d) F_{n-1} .
\end{aligned}
$$

From (25), (26) we have

$$
{ }^{(1)} f_{8} \leqq F_{s-1} \leqq F_{n-2}<(m: d) F_{n-1}^{\prime} \text {. }
$$

(B) follows from (22). Thus (29) is proved.

$$
\left[g_{n-s, t}\right]=-1+\bar{g}_{n-s, t} . \quad(s=2, \cdots, n ; t=1, \cdots, s-1)
$$

Proof of (30). We have to prove
(A) $-1+\bar{g}_{n-8, t}<g_{n-8, t}$;
(B) $g_{n-8, t}<\bar{g}_{n-8, t} \cdot$

To prove (A) we have to show

$$
\begin{aligned}
\bar{g}_{n-8, t}-g_{n-8, t} & <1, \quad \text { or, dividing by } D-w, \\
{ }^{(1)} g_{n-8, t} & <(m: d) F_{n-1} .
\end{aligned}
$$

But from (27) we have

$$
{ }^{(1)} g_{n-8, t}<(m: d) F_{n-8+t-1} \leqq(m: d) F_{n-2}<(m: d) F_{n-1} \text {. }
$$

To prove (B) we have to show, after dividing by $D-w$

$$
{ }^{(1)} g_{n-8, t}>0 \text {. }
$$


But for $s<n$

$$
{ }^{(1)} g_{n-s, t} \geqq{ }^{(1)} g_{n-s, t}-{ }^{(1)} g_{n-s, t-1}=g_{n-(s+1), t}>0 \text {; }
$$

and for $s=n$

$$
{ }^{(1)} g_{0, t} \geqq{ }^{(1)} g_{0, t}-{ }^{(1)} g_{0, t-1}=(m: d) f_{t-1}>0,
$$

(that the expressions $g_{n-s, t}$ are positive entities will become clear ${ }_{2}$ later, while carrying out the JAPAL for the $f_{i}$ ).

$$
\frac{f_{i}-\bar{f}_{i}+1}{f_{j}-\bar{f}_{j}+1}<1 . \quad(j<i<n-1 ; j=1, \cdots, n-2) .
$$

Proof of (31). It was shown that the denominator is positive. We therefore have to prove, after dividing by $D-w$

$$
{ }^{(1)} f_{i}-{ }^{(1)} f_{j}>0 \text {, }
$$

which follows directly from (17a).

$$
\frac{(D-w) f_{j}}{f_{s}-\bar{f}_{s}+1}<1 . \quad(j=0,1, \cdots, s-2 ; s=2,3, \cdots, n-1) .
$$

Proof of (32). We have to show

$$
\begin{aligned}
(D-w) f_{j} & <f_{s}-\bar{f}_{s}+1, \quad \text { or, dividing by } D-w, \\
f_{j}+{ }^{(1)} f_{s} & <(m: d) F_{n-1} .
\end{aligned}
$$

But

$$
\begin{aligned}
f_{j}+{ }^{(1)} f_{s}<f_{s-2}+F_{s-1}<F_{n-3}+F_{n-2}<2 F_{n-2} & <(m: d) F_{n-1} . \\
1<\frac{f_{i-1}-\bar{f}_{i-1}+1}{f_{i}-\bar{f}_{i}+1}<2 . & (i=1, \cdots, n-1) .
\end{aligned}
$$

Proof of (33). We have to prove, since the left hand inequality was proved in (31)

$$
f_{i-1}-\bar{f}_{i-1}+1<2\left(f_{i}-\bar{f}_{i}\right)+2,
$$

or carrying over and dividing $m$ by $D-w$

$$
2 \cdot{ }^{(1)} f_{i}-{ }^{(1)} f_{i-1}<(m: d) F_{n-1} \text {. }
$$

But

$$
\begin{aligned}
& \quad 2 \cdot{ }^{(1)} f_{i}-{ }^{(1)} f_{i-1} \leqq 2 \cdot{ }^{(1)} f_{i} \leqq 2 F_{n-2}<\frac{m}{d} F_{n-1} . \\
& \frac{g_{n-s . t}-\bar{g}_{n-8, t}+1}{f_{q}-\bar{f}_{q}+1}<1 . \\
& \quad(s=2,3, \cdots, n ; t=1,2, \cdots, s-1 ; q=1,2, \cdots, n-s) .
\end{aligned}
$$


Proof of (34). We have to prove

$$
g_{n-s, t}-\bar{g}_{n-s, t}+1<f_{q}-\bar{f}_{q}+1,
$$

or, dividing by $D-w$,

$$
{ }^{(1)} g_{n-8, t}-{ }^{(1)} f_{q}>0,
$$

which follows directly from (20e).

$$
\frac{g_{n-8, t}-\bar{g}_{n-s, t}+1^{2)}}{g_{n-s, j}-\bar{g}_{n-8, j}+1}<1 . \quad\left(1 \leqq j<t_{-} \leqq s-1 \leqq n-1\right)
$$

Proof of (35). We have to prove

$$
g_{n-8, t}-\bar{g}_{n-8, t}+1<g_{n-8, j}-\bar{g}_{n-8, j}+1 \text {, }
$$

or, after carrying over and dividing by $D-w$

$$
{ }^{(1)} g_{n-8, t}-{ }^{(1)} g_{n-8, j}>0 \text {, }
$$

which follows directly from (20a).

$$
1<\frac{f_{n-8}-\bar{f}_{n-s}+1}{g_{n-s, 1}-\bar{g}_{n-s, 1}+1}<2 . \quad(s=2,3, \cdots, n) .
$$

Proof of (36). We have to prove

$$
\begin{aligned}
& \text { (A) } g_{n-8,1}-\bar{g}_{n-8,1}+1<f_{n-8}-\bar{f}_{n-s}+1 \text {, } \\
& \text { (B) } f_{n-8}-\bar{f}_{n-8}+1<2\left(g_{n-8,1}-\bar{g}_{n-8,1}\right)+2 .
\end{aligned}
$$

To prove (A) we have to show, after carrying over and dividing by $D-w$

$$
{ }^{(1)} g_{n-8,1}-{ }^{(1)} f_{n-8}>0
$$

But from (19) we have

$$
{ }^{(1)} g_{n-8,1}-{ }^{(1)} f_{n-8}=g_{n-(8+1), 1}, \quad \text { for } s<n .
$$

For $n=s$ we have

$$
{ }^{(1)} g_{0,1}-{ }^{(1)} g_{0,0}=(m: d) f_{0}>1 \text {. }
$$

To prove (B) we have to show, after carrying over and dividing by $D-w$

$$
2 \cdot{ }^{(1)} g_{n-8,1}-{ }^{(1)} f_{n-8}<(m: d) F_{n-1} \text {. }
$$

But

$$
\begin{aligned}
& 2 \cdot{ }^{(1)} g_{n-8,1}-{ }^{(1)} f_{n-s} \leqq 2 \cdot{ }^{(1)} g_{n-8,1}<2 F_{n-8} \leqq 2 F_{n-2}<(m: d) F_{n-1} \\
& \frac{(D-w) f_{r-1}}{g_{n-8, t}-\bar{g}_{n-8, t}+1}<1 . \\
& \quad(r=1,2, \cdots, n-s ; s=2,3, \cdots, n-1 ; t=1,2, \cdots, s-1) .
\end{aligned}
$$

${ }^{2}$ While carrying out the JAPAL in the following chapter, it will become clear that the numerator and denominator are positive entities. 
Proof of (37). We have to prove

$$
(D-w) f_{r-1}<g_{n-8, t}-\bar{g}_{n-s, t}+1,
$$

or, after carrying over and dividing by $D-w$

$$
{ }^{(1)} g_{n-8, t}+f_{r-1}<(m: d) F_{n-1} \text {, }
$$

or a fortiori

$$
\begin{aligned}
& { }^{(1)} g_{n-s, t}+f_{r-1}<F_{n-s+t-1}+F_{r-1}<2 F_{n-2}<(m: d) F_{n-1} \cdot \\
& 1<\frac{g_{n-s, t}-\bar{g}_{n-s, t}+1}{g_{n-s, t+1}-\bar{g}_{n-s, t+1}+1}<2 . \\
& \quad(s=2,3, \cdots, n ; t=1,2, \cdots, s-2) .
\end{aligned}
$$

Proof of (38). We have to prove

(A) $g_{n-s, t}-\bar{g}_{n-s, t}+1>g_{n-s, t+1}-\bar{g}_{n-s, t+1}+1$,

(B) $g_{n-s, t}-\bar{g}_{n-s, t}+1<2\left(g_{n-s, t+1}-\bar{g}_{n-s, t+1)}+2\right.$.

To prove (A) we have to show, after carrying over and dividing by $D-w$

$$
{ }^{(1)} g_{n-s, t+1}-{ }^{(1)} g_{n-s, t}>0 \text {, }
$$

which follows from

$$
{ }^{(1)} g_{n-8, t+1}-{ }^{(1)} g_{n-s, t}=g_{n-(s+1), t+1}>0
$$

for $s \leqq n-1$. For $s=n$ the proof is exactly as before.

To prove (B) we have to show, after carrying over and dividing by $D-w$

$$
2 \cdot{ }^{(1)} g_{n-s, t+1}-{ }^{(1)} g_{n-s, t}<(m: d) F_{n-1},
$$

which follows from

$$
\begin{aligned}
2 \cdot{ }^{(1)} g_{n-s, t+1}-{ }^{(1)} g_{n-s, t} & \leqq 2 \cdot{ }^{(1)} g_{n-s, t+1} \\
& <2 F_{n-s+t} \leqq 2 F_{n-2} \leqq(m: d) F_{n-1}
\end{aligned}
$$

V. The JAPAL of the $f_{1}, f_{2}, \cdots, f_{n-1}$. We shall now carry out the JAPAL of the numbers $f_{1}, f_{2}, \cdots, f_{n-1}$ and thus complete the proof of the Main Theorem. To this end I shall introduce still a few more new conceptions.

Definition 4. The set of $n-1$ numbers $a_{i}^{(v)}(i=1, \cdots, n-1$; $v=0,1, \cdots)$ shall be called the $v$ th generator of the JAPAL, the number $a_{i}^{(v)}$ its $i$ th element; the set of $n-1$ numbers

$$
b_{i}^{(v)}=\left[a_{i}^{(v)}\right]
$$


( $i, v$ as before) shall be called the $v$ th genus of the JAPAL, $b_{i}^{(v)}$ its $i$ th element. The key to the final proof of the Main Theorem now rests with the

LEMMA. Let the $n-1$ elements of the vth generator fulfill the following conditions:

(A) $\quad a_{i}^{(v)}=P_{i}(w, D) ; \quad P_{1}(w, D)=w+c_{1} D ; \quad P_{0}(w, D)=\bar{P}_{0}(w, D)=1 ;$

$$
\begin{aligned}
& {\left[\alpha_{i}^{(v)}\right]=-1+\bar{P}_{i}(w, D) .} \\
& \quad\left(i=1,2, \cdots, n-1 ; c_{1} \text { a nonnegative integer }\right) \\
& 0<\frac{P_{t+k}-\bar{P}_{t+k}+1}{P_{t}-\bar{P}_{t}+1}<1 \\
& \quad(t=1, \cdots, n-2 ; k=1, \cdots, n-2 ; t+k \leqq n-1) \\
& 0<\frac{(D-w)\left({ }^{(1)} P_{q-1}-{ }^{(1)} P_{q-2}\right)}{P_{t}-\bar{P}_{t}+1}<1 ; \\
& \quad(q=2,3, \cdots, t ; t=2,3, \cdots, n-1) \\
& 1<\frac{P_{t-1}-\bar{P}_{t-1}+1}{P_{t}-\bar{P}_{t}+1}<2 . \quad(t=1, \cdots, n-1) .
\end{aligned}
$$

Then the $n$ genera

$$
b_{1}^{(v+k)}, b_{2}^{(v+k)}, \cdots, b_{n-1}^{(v+k)} \quad(k=0,1, \cdots, n-1)
$$

form a fugue, and the elements of the $v+n$th generator, namely the $a_{i}^{(v+n)}(i=1,2, \cdots, n-1)$ have the form

$$
\begin{array}{ll}
a_{i}^{(v+n)}={ }^{(1)} P_{i+1}-{ }^{(1)} P_{i} ; & (i=1, \cdots, n-2) \\
a_{n-1}^{(v+n)}=\frac{P_{n-1}-\bar{P}_{n-1}+1}{D-w} . &
\end{array}
$$

Proof of the lemma. In view of (39) (A), (B) and following formula (1) the elements of the $v+1$ st generator have the form

$$
\begin{array}{ll}
a_{i}^{(v+1)}=\frac{P_{i+1}-\bar{P}_{i+1}+1}{P_{1}-\bar{P}_{1}+1}, \quad(i=1, \cdots, n-2) ; \\
a_{n-1}^{(v+n)}=\frac{1}{P_{1}-\bar{P}_{1}+1} .
\end{array}
$$

Since the elements of the $v+1$ st generator fulfill the conditions (39) (C), the elements of the $v+1$ st genus have the form

$$
b_{i}^{(v+1)}=0 ; \quad(i=1,2, \cdots, n-2) \quad b_{n-1}^{(v+1)}=1 .
$$

On the basis of (40a), (40b) and reminding from (39) that $\bar{P}_{1}-P_{1}=$ $(D-w)=(D-w)\left({ }^{(1)} P_{1}-{ }^{(1)} P_{0}\right)$ we obtain, following (1), for the $n-1$ 
elements of the $v+2$ nd generator

$(40 c)$

$$
\begin{aligned}
& a_{i}^{(v+2)}=\frac{P_{i+2}-\bar{P}_{i+2}+1}{P_{2}-\bar{P}_{2}+1} ; \quad(i=1,2, \cdots, n-3) \\
& a_{n-2}^{(v+2)}=\frac{(D-w)\left({ }^{(1)} P_{1}-{ }^{(1)} P_{0}\right)}{P_{2}-\bar{P}_{2}+1}, \\
& a_{n-1}^{(v+2)}=\frac{P_{1}-\bar{P}_{1}+1}{P_{2}-\bar{P}_{2}+1} .
\end{aligned}
$$

Now the elements $a_{i}^{(v+2)}(i=1,2, \cdots, n-1)$ again satisfy conditions (39) (C), and therefore the elements of the $v+2$ nd genus have the form

$$
b_{i}^{(v+2)}=0 ; \quad(i=1, \cdots, n-2) \quad b_{n-1}^{(v+2)}=1 .
$$

In view of $(40 \mathrm{c}),(40 \mathrm{~d})$ and (1) the elements of the $v+3 \mathrm{rd}$ generator have the form

$(40 \mathrm{e})$

$$
\begin{aligned}
& a_{i}^{(v+3)}=\frac{P_{i+3}-\bar{P}_{i+3}+1}{P_{3}-\bar{P}_{3}+1}, \quad(i=1,2, \cdots, n-4) \\
& a_{n-3}^{(v+3)}=\frac{(D-w)\left({ }^{(1)} P_{1}-{ }^{(1)} P_{0}\right)}{P_{3}-\bar{P}_{3}+1}, \\
& a_{n-2}^{(v+3)}=\frac{(D-w)\left({ }^{(1)} P_{2}-{ }^{(1)} P_{1}\right)}{P_{3}-\bar{P}_{3}+1}, \\
& a_{n-1}^{(v+3)}=\frac{P_{2}-\bar{P}_{2}+1}{P_{3}-\bar{P}_{3}+1} .
\end{aligned}
$$

Continuing these considerations one arrives quite easily and by induction at the conclusion that the $v+t$ th generator takes the form

(40f)

$$
\begin{array}{rlrl}
a_{i}^{(v+t)} & =\frac{P_{i+t}-\bar{P}_{i+t}+1}{P_{t}-\bar{P}_{t}+1}, & (i=1, \cdots, n-t-1) \\
a_{n-t-1-j}^{(v+t)} & =\frac{(D-w)\left({ }^{(1)} P_{j}-{ }^{(1)} P_{j-1}\right)}{P_{t}-\bar{P}_{t}+1}, & & (j=1, \cdots, t-1) \\
a_{n-1}^{(v+t)} & =\frac{P_{t-1}-\bar{P}_{t-1}+1}{P_{t}-\bar{P}_{t}+1}, &
\end{array}
$$

and that the $n-1$ elements of the $v+t$ th genus have the form

$$
b_{i}^{(v+t)}=0 ; \quad(i=1, \cdots, n-2) \quad b_{n-1}^{(v+t)}=1 .
$$

Following the formulas (40f), $(40 \mathrm{~g})$ and (1) we obtain that the $n-1$ elements of the $v+n-1$ st generator have the form

$$
\begin{aligned}
& a_{i}^{(v+n-1)}=\frac{(D-w)\left({ }^{(1)} P_{i}-{ }^{(1)} P_{i-1}\right)}{P_{n-1}-\bar{P}_{n-1}+1}, \quad(i=1, \cdots, n-2) \\
& a_{n-1}^{(v+n-1)}=\frac{P_{n-2}-\bar{P}_{n-2}+1}{P_{n-1}-\bar{P}_{n-1}+1}
\end{aligned}
$$


and that, on ground of (39) (C) the elements of the $v+n-1$ st genus have the form

$$
b_{i}^{(v+n-1)}=0 ; \quad(i=1,2, \cdots, n-2) \quad b_{n-1}^{(v+n-1)}=1 .
$$

Thus the $n$ genera

$$
b_{1}^{(v+j)}, b_{2}^{(v+j)}, \cdots, b_{n-1}^{(v+j)}, \quad(j=0,1, \cdots, n-1)
$$

indeed form a fugue as was stated in the lemma. Now we have from (40h)

$$
a_{1}^{(v+n-1)}=\frac{(D-w)\left(^{(1)} P_{1}-{ }^{(1)} P_{0}\right)}{P_{n-1}-\bar{P}_{n-1}+1}=\frac{D-w}{P_{n-1}-\bar{P}_{n-1}+1},
$$

so that on the basis of $(40 \mathrm{~h}),(40 \mathrm{i}),(40 \mathrm{j})$ we receive for the $n-1$ elements of the $v+n$th generator

$$
\begin{aligned}
& a_{i}^{(v+n)}={ }^{(1)} P_{i+1}-{ }^{(1)} P_{i}, \quad(i=1,2, \cdots, n-2) \\
& a_{n-1}^{(v+n)}=\frac{P_{n-1}+\bar{P}_{n-1}+1}{D-w} .
\end{aligned}
$$

By this the lemma is completely proved.

We are now able to prove the main Theorem quite easily in the following steps:

(1) Let be

$$
P_{i}(w, D)=f_{i}(w, D)=a_{i}^{(0)} . \quad(i=1,2, \cdots, n-1) .
$$

Following (29), (31), (32), (33) the functions $f_{i}(i=1, \cdots, n-1)$ indeed fulfill the conditions (39) (A), (B), (C). Therefore, following the lemma, we get for the $n+1$ st generator, which is the first generator of the second fugue of the JAPAL

$$
\begin{aligned}
& a_{i}^{(n)}={ }^{(1)} f_{i+1}-{ }^{(1)} f_{i}, \quad(i=1,2, \cdots, n-2) \\
& a_{n-1}^{(n)}=\frac{f_{n-1}-\bar{f}_{n-1}+1}{D-w} \text {, }
\end{aligned}
$$

so that on the ground of

$$
\begin{aligned}
\frac{f_{n-1}-\bar{f}_{n-1}+1}{D-w} & =\frac{1}{D-w}-{ }^{(1)} f_{n-1} \\
& =(m: d) F_{n-1}-\sum_{i=0}^{n-2}(-1)^{i} F_{n-2-i} \\
& =\sum_{i=0 / m: d}^{0 / n-1}(-1)^{i} F_{n-1-i}=g_{n-2,1}
\end{aligned}
$$

and on the basis of (17) we have for the $n-1$ elements of the first 

generator of the second fugue of the JAPAL

$$
a_{i}^{(n)}=f_{i} ; \quad(i=1,2, \cdots, n-2) a_{n-1}^{(n)}=g_{n-2,1} .
$$

(2) Let

$$
P_{i}(w, D)=f_{i}
$$$$
(i=1,2, \cdots, n-2)
$$

2) $P_{n-1}(w, D)=g_{n-2,1}$.

Following the formulas (29) to (37) the functions of (41b) fulfill the conditions (39), and therefore the elements of the first generator of the third fugue have the form

$$
\begin{array}{lr}
a_{i}^{(2 n)}={ }^{(1)} f_{i+1}-{ }^{(1)} f_{i} ; & (i=1,2, \cdots, n-3) \\
a_{n-2}^{(2 n)}={ }^{(1)} g_{n-2,1}-{ }^{(1)} f_{n-2} ; & a_{n-1}^{(2 n)}=\frac{g_{n-2,1}-\bar{g}_{n-2,1}+1}{D-w} .
\end{array}
$$

Following the formulas (17), (19), (20c) we get for the functions (42)

$$
\begin{array}{ll}
a_{i}^{(2 n)}=f_{i} ; & (i=1,2, \cdots, n-3) \\
a_{n-2}^{(2 n)}=g_{n-3,1} ; & a_{n-1}^{(2 n)}=g_{n-3,2} .
\end{array}
$$

In the same way we get from (42a) that the $n-1$ elements of the first generator of the fourth fugue have the form

$$
\begin{aligned}
& a_{i}^{(3 n)}=f_{i} \text {; } \\
& (i=1,2, \cdots, n-4) \quad a_{n-3}^{(3 n)}=g_{n-4,1} ; \\
& a_{n-2}^{(3 n)}=g_{n-4,2} ; \quad a_{n-1}^{(3 n)}=g_{n-4,3} \text {. }
\end{aligned}
$$

Continuing this process of the JAPAL we get from (43) that the elements of the first generator of the $s$ th fugue have the form $(s=$ $2,3, \cdots, n)$

$$
\begin{array}{ll}
a_{i}^{((s-1) n)}=f_{i} ; & (i=1,2, \cdots, n-s) \\
a_{n-s+t}^{((s-1) n)}=g_{n-s, t} . & (t=1,2, \cdots, s-1) .
\end{array}
$$

From (44) we finally deduce, for $n=s$, that the elements of the first generator of the $n$th fugue have the form

$$
a_{i}^{((n-1) n)}=g_{0, t} . \quad(t=1,2, \cdots, n-1) .
$$

But we have from (11)

$$
\begin{aligned}
g_{n-n, 1} & =g_{0,1}=\sum_{i=0 / m: d}^{0 / 1}(-1)^{i}\left(\begin{array}{c}
n-2+i \\
i
\end{array}\right) F_{1-i} \\
& =(m: d) F_{1}-(n-1) F_{0}=(m: d) F_{1}-(n-1),
\end{aligned}
$$

so that

$$
\bar{g}_{0,1}-g_{0,1}=(m: d)\left(\bar{F}_{1}-F_{1}\right)=(m: d)(D-w) \text {. }
$$

With this and on the basis of the lemma, we get from (45) that the 
elements of the first generator of the $n+1$ st fugue have the form

$$
\begin{array}{ll}
a_{i}^{\left(n^{2}\right)}=\left({ }^{(1)} g_{0, i+1}-{ }^{(1)} g_{0, i}\right)(m: d) ; & (i=1, \cdots, n-2) \\
a_{n-1}^{\left(n^{2}\right)}=\frac{g_{0, n-1}-\bar{g}_{0, n-1}+1}{(m: d)(D-w)} . &
\end{array}
$$

Now according to $(20 \mathrm{~b}),(20 \mathrm{~d})$ we have

$$
a_{i}^{\left(n^{2}\right)}=f_{i} ; \quad(i=1,2, \cdots, n-2) \quad a_{n-1}^{\left(n^{2}\right)}=f_{n-1} .
$$

From (41) and (46a) we have

$$
a_{i}^{\left(n^{2}\right)}=a_{i}^{(0)}, \quad(i=1,2, \cdots, n-1)
$$

so that the $n-1$ elements of the first generator of the first fugue are identical with the $n-1$ elements of the first generator of the $n+1$ st fugue. Thus (47) shows that the JAPAL of the $f_{i}(i=$ $1,2, \cdots, n-1)$ is purely periodic with the length $n^{2}$ ( $n$ fugues), as stated by the Main Theorem.

Now since

$$
\begin{aligned}
& \bar{f}_{i}=\sum_{i=0}^{s}\left(\begin{array}{c}
n-1-s+i \\
i
\end{array}\right) D^{s-i}(D-1)^{i} . \quad(i=1, \cdots, n-1) \\
& \bar{g}_{n-s, t}=\sum_{i=0 / m: d}^{t-1 / n-s+t}(-1)^{i}\left(\begin{array}{c}
s-t+i \\
i
\end{array}\right) \bar{F}_{n-s+t-i} \\
& =\sum_{i=0 / m: d}^{t-1 / n-s+t}(-1)^{i}\left(\begin{array}{c}
s-t+i \\
i
\end{array}\right)\left(\begin{array}{c}
n \\
s-t+i
\end{array}\right) D^{n-s+t-i}, \\
& (s=2,3, \cdots, n ; t=1,2, \cdots, s-1),
\end{aligned}
$$

and since we have for the elements of the various genera of the JAPAL either

$$
-1+\bar{f}_{i} \quad \text { or } \quad-1+\bar{g}_{n-8, t}
$$

the pattern of the accumulators of the $n$ fugues of the JAPAL as indicated in the formulas (6) to (6d) becomes immediately obvious. If $m=d=1$ we have

$$
g_{n-2,1}=\sum_{i=0}^{0 / n-1}(-1)^{i} F_{n-1-i}=\sum_{i=0}^{n-1}(-1)^{i} F_{n-1-i}=f_{n-1} .
$$

We therefore get from (41a) that in this case the elements of the first generator of the second fugue have the form

$$
a_{i}^{(n)}=f_{i}, \quad(i=1,2, \cdots, n-1)
$$

so that here 
NEW INFINITE CLASSES OF PERIODIC JACOBI-PERRON ALGORITHMS 465

$$
a_{i}^{(u)}=a_{i}^{(0)},
$$$$
(i=1,2, \cdots, n-1)
$$

as stated in the Main Theorem, which, through this final remark, is completely proved.

Proof of Corollary 1. We make the following substitutions in $w=\left(D^{n}-d: m\right)^{1: n}:$ Let $T, t$ be natural numbers such that $t \mid T$, $t \geqq 1$, let denote

$$
D=T: t ; \quad d=1 ; \quad m=t^{n-k} . \quad(k=0,1, \cdots, n) .
$$

Following the conditions of the Main Theorem, we have here

$$
1 \leqq t \leqq T: 2(n-1)
$$

Further $w$ takes the form

$$
w=W: t ; \quad W=\left(T^{n}-t^{k}\right)^{1: n} .
$$

The functions $f_{s}, F_{s}, g_{n-s, t}$ take the form

$$
\begin{aligned}
& f_{s}=t^{-s} \sum_{i=0}^{s}\left(\begin{array}{c}
n-1-s+i \\
i
\end{array}\right) W^{s-i}(T-t)^{i} ; \quad(s=1, \cdots, n-1) \\
& F_{s}=t^{-s} \sum_{i=0}^{s}\left(\begin{array}{c}
n-1-s+i \\
i
\end{array}\right) W^{s-i} T^{i} ; \quad(s=1, \cdots, n-1) \\
& g_{n-s, u}=\sum_{i=0 / t n-k}^{u-1 / n-s+u}(-1)^{i}\left(\begin{array}{c}
s-u-1+i \\
i
\end{array}\right) F_{n-s+u-i} \\
&(s=2,3, \cdots, n ; u=1,2, \cdots, s-1) .
\end{aligned}
$$

If we substitute again in (49a), (49b)

$$
D \text { for } T ; \quad d \text { for } t ; \quad w \text { for } W \text {, }
$$

we get from the Main Theorem, that the JAPAL of the $n-1$ numbers

$$
\begin{aligned}
& f_{s}=d^{-s} \sum_{i=0}^{s}\left(\begin{array}{c}
n-1-s+i \\
i
\end{array}\right) w^{s-i}(D-d)^{i}, \quad w=\left(D^{n}-d^{k}\right)^{1: n} \\
& (s=1,2, \cdots, n-1 ; k=0,1, \cdots, n)
\end{aligned}
$$

takes the form as indicated in Corollary 1.

Proof of Corollary 2. Here we make the following substitutions in $w$. Let $T, t$ be natural numbers, $t \mid T$; let

$$
D=T: t ; \quad d=1 ; \quad m=t^{n-r}: T . \quad(r=0,1, \cdots, n-2) .
$$

The reader should note that the condition that $m$ is a natural number is necessary only for the purpose that the elements of the 
accumulators in (6c), (6d) be integers. For the proof of the Main Theorem we made use only of the fact that $m \geqq 1$. The elements of the accumulators in (6c), (6d) may be integers even if $m$ is not an integer, as was proved at the end of Chapter II. From $1 \leqq m=t^{n-r}: T$, we derive

$$
T \leqq t^{n-r},
$$

and from $1 \leqq D: 2 d(n-1)$ and (50a)

$$
2 t(n-1) \leqq T \leqq t^{n-r} ; \quad t \geqq(2(n-1))^{1:(n-r-1)} .
$$

From $t \mid T$ and (50a) we derive the condition of (50), namely $r=$ $0,1, \cdots, n-2$. For $r=n-2$ we have $T=t^{2} . \quad w$ takes the form

$$
w=W: t ; \quad W=\left(T^{n}-t^{r} T\right)^{1: n} . \quad(r=0, \cdots, n-2) .
$$

If we again substitute

$$
D \text { for } T ; d \text { for } t ; w \text { for } W
$$

and follow the proof of Corollary 1, the proof of Corollary 2 will be completed.

Corollary 3. Let $d, D, u, m$ be natural numbers and $p$ a prime number such that

$$
d \mid D ; \quad u, m \geqq 1 ; \quad d p \leqq D: 2\left(p^{u}-1\right),
$$

and let denote

$$
\begin{aligned}
& w=\left(D^{p u}-p d: m\right)^{p-u}, \\
& f_{s}(w, D-1)=\sum_{i=0}^{s}\left(\begin{array}{c}
n-1-s+i \\
i
\end{array}\right) w^{s-i}(D-1)^{i} . \\
&(s=1, \cdots, n-1) .
\end{aligned}
$$

Then the JAPAL of the $n-1$ numbers

$$
f_{1}(w, D-1), f_{2}(w, D-1), \cdots, f_{\left(p^{u}-1\right)}(w, D-1)
$$

is purely periodic and its primitive length is $p^{2 u}$. The period consists of $p^{u}$ fugues, each fugue being a matrix of $p^{u}$ rows and $p^{u}-1$ columns. The accumulators of the fugues have the form as those in the Main Theorem, namely (6) to (6d), where $d$ is substituted by pd and $n$ by $p^{u}$.

Proof of Corollary 3. All we have to prove is to show that all those integers which appear in the accumulators and are multiples of $d$ are also multiples of $p$. This concerns all the numbers 
(52a)

$$
\begin{aligned}
& (-1)^{i}\left(\begin{array}{c}
s-1-t+i \\
i
\end{array}\right)\left(\begin{array}{c}
p^{u} \\
s-t+i
\end{array}\right) D^{n-s+t-i}, \\
& \left(i=0,1, \cdots, t-1 ; t=1,2, \cdots, s-1 ; s=2,3, \cdots, p^{u}\right)
\end{aligned}
$$

where the decisive point is the relation

$$
1 \leqq s-t+i \leqq p^{u}-1
$$

But since, as is well known,

$$
p \mid\left(\begin{array}{l}
p^{u} \\
k
\end{array}\right) \quad \text { for } u=1,2, \cdots ; k=1,2, \cdots, p^{u}-1
$$

it follows from $(52 c)$ in view of $(52 b)$ that the numbers in (52a) are all multiples of $p$.

We leave it to the reader to prove the interesting fact, that each element of all the accumulators (6) to (6d) appearing in the Main Theorem are multiples of $p$, if $n=p^{u}$ ( $p$ prime, $\left.u=1,2, \cdots\right)$

VI. Illustrations. (1) To illustrate the Main Theorem let us take $n=5$. Then the Main Theorem would sound:

Let $d, D, m$ be natural numbers such that

$$
d \mid D ; \quad m \geqq 1 ; \quad 1 \leqq d \leqq D: 8 .
$$

Let

$$
\begin{aligned}
& w=\left(D^{s}-d: m\right)^{1: 5}, \\
& f_{s}=\sum_{i=0}^{s}\left(\begin{array}{c}
4-s+i \\
i
\end{array}\right) w^{s-i}(D-1)^{i} . \quad(s=1,2,3,4) .
\end{aligned}
$$

Then the JAPAL of the 4 numbers

$$
\begin{aligned}
& w+4(D-1) ; \quad w^{2}+3 w(D-1)+6(D-1)^{2} ; \\
& w^{3}+2 w^{2}(D-1)+3 w(D-1)^{2}+4(D-1)^{3} ; \\
& w^{4}+w^{3}(D-1)+w^{2}(D-1)^{2}+w(D-1)^{3}+(D-1)^{4}
\end{aligned}
$$

is purely periodic and its primitive length is 25 . The period consists of five fugues, and the accumulators of these fugues have the form:

First fugue

$$
\begin{aligned}
& 5(D-1) ; \quad 5(D-1)(2 D-1) ; \quad 5(D-1)\left(2 D^{2}-2 D+1\right) ; \\
& 5 D(D-1)\left(D^{2}-D+1\right) ;
\end{aligned}
$$

Second fugue 


$$
\begin{aligned}
& 5(D-1) ; \quad 5(D-1)(2 D-1) ; \quad 5(D-1)\left(2 D^{2}-2 D+1\right) ; \\
& 5 D\left(\frac{m D^{3}}{d}-2 D^{2}+2 D-1\right) ;
\end{aligned}
$$

Third fugue

$$
\begin{aligned}
& 5(D-1) ; \quad 5(D-1)(2 D-1) ; \quad 5 D\left(\frac{2 m D^{3}}{d}-4 D^{2}+3 D-1\right) ; \\
& 5 D\left(\frac{m D^{2}}{d}(D-2)+2 D-1\right) ;
\end{aligned}
$$

Fourth fugue

$$
\begin{aligned}
& 5(D-1) ; 5\left(\frac{2 m D^{2}}{d}-3 D+1\right) ; 5\left(\frac{2 m D^{2}}{d}(D-2)\right)+15 D-5, \\
& 5 D\left(\frac{m D}{d}\left(D^{2}-2 D+2\right)-1\right) ;
\end{aligned}
$$

Fifth fugue

$$
\begin{aligned}
& 5\left(\frac{m D}{d}-1\right) ; 5\left(\frac{m D}{d}(2 D-3)+1\right) ; 5 \frac{m D}{d}\left(\left(2 D^{2}-4 D+3\right)-1\right) ; \\
& \frac{5 m D}{d}\left(D^{3}-2 D^{2}+2 D-1\right) .
\end{aligned}
$$

In the case of $n=5, m=d=1$, the JAPAL of the 4 numbers

$$
f_{8}=\sum_{i=0}^{s}\left(\begin{array}{c}
4-s+i \\
i
\end{array}\right) w^{s-i}(D-1)^{i} ; \quad(s=1,2,3,4) w=\left(D^{s}-1\right)^{1: 5}
$$

is purely periodic and its primitive length is 5 . It consists of one fugue, the accumulator of which has the form

$$
\begin{aligned}
& 5(D-1) ; \quad 5(D-1)(2 D-1) ; \quad 5(D-1)\left(2 D^{2}-2 D+1\right) ; \\
& 5 D(D-1)\left(D^{2}-D+1\right) .
\end{aligned}
$$

To illustrate Corollary 3 we shall take $p=2 ; u=2$. Then Corollary 3 would sound:

Let $d, D$ be natural numbers such that $d \mid D, d \leqq D: 12$ and let $w=\left(D^{4}-2 d\right)$; then the JAPAL of the three numbers

$$
\begin{aligned}
& w+3(D-1) ; \quad w^{2}+2 w(D-1)+3(D-1)^{2} ; \\
& w^{3}+w^{2}(D-1)+w(D-1)^{2}+(D-1)^{3}
\end{aligned}
$$

is purely periodic and its primitive length is 16 . The period consists of four fugues, the accumulators of which have the form 
First fugue

$$
4(D-1) ; \quad 2(D-1)(3 D-1) ; \quad 2(D-1)\left(2 D^{2}-D+1\right) ;
$$

Second fugue

$$
4(D-1) ; \quad 2(D-1)(3 D-1) ; \quad 2\left(\frac{m D^{3}}{d}-3 D^{2}+2 D-1\right) ;
$$

Third fugue

$$
4(D-1) ; \quad \frac{3 m D^{2}}{d}-8 D+2 ; \quad \frac{m D^{2}}{d}(2 D-3)+2(2 D-1) ;
$$

Fourth fugue

$$
2\left(\frac{m D}{d}-2\right) ; \quad \frac{m D}{d}(3 D-4)+2 ; \quad \frac{m D}{d}\left(2 D^{2}-3 D+2\right)-2 .
$$

\section{REFERENCES}

1. C. G. J. Jacobi, Allgemeine Theorie der kettenbruchaehnlichen Algorithmen, in welche jede Zahl aus drei vorhergehenden gebildet wird, Journal f. d. reine und angew. Math. 69 (1868), 29-64.

2. Oskar Perron, Grundlagen fuer eine Theorie des Jacobischen Kettenbruchalgorithmus, Math. Annalen 64 (1907), 1-76.

3. Leon Bernstein, Periodical continued fractions of irrationals of degree $n$ by Jacobi's Algorithm, Journal f.d. reine und angew. Math. Band 213, Heft 1/2, 1963.

4. - Periodicity of Jacobi's algorithm for a special type of cubic irrationals, Journal f. d. reine und angew. Math. Band 214, Heft 1/2, 1964.

5. - Periodische Jacobische algorithmen fuer eine unendliche Klasse Algebraischer Irrationalzahlen, Journal f.d. reine und angew. Math., Band 214, Heft 3/4, 1964.

6. The Continued Fraction of $\left(D^{n}-d\right)^{1: n}$, Mathematische Nachrichten, to appear soon.

7. — Periodische Jacobi-Perronsche Algorithmen, Archiv der Mathematik, to appear soon,

8. - Rational approximation of algebraic irrationals by means of a modified Jacobi algorithm, Duke Math. J., to appear soon.

9. —_ Periodic Jacobi-Perron algorithm for numbers of the algebraic field $K(x)$, $x$ a root of the general equation of degree $n$, Canad. J. Math., to appear soon.

10. - and Helmut Hasse, Einheitsbestimmung mittels des Jacobi-Perronschen Algorithmus, Journal f.d. reine und angew. Math., to appear soon. 



\section{PACIFIC JOURNAL OF MATHEMATICS}

\section{EDITORS}

\author{
H. SAMmLSON \\ Stanford University \\ Stanford, California \\ R. M. BLUMENTHAL \\ University of Washington \\ Seattle, Washington 98105
}

\author{
*J. DUGUNDJI \\ University of Southern California \\ Los Angeles, California 90007
}

RICHARD ARENS

University of California

Los Angeles, California 90024

\section{E. F. BECKENBACH \\ B. H. NEUMANN \\ ASSOCIATE EDITORS}

\section{SUPPORTING INSTITUTIONS}

\author{
UNIVERSITY OF BRITISH COLUMBIA \\ CALIFORNIA INSTITUTE OF TECHNOLOGY \\ UNIVERSITY OF CALIFORNIA \\ MONTANA STATE UNIVERSITY \\ UNIVERSITY OF NEVADA \\ NEW MEXICO STATE UNIVERSITY \\ OREGON STATE UNIVERSITY \\ UNIVERSITY OF OREGON \\ OSAKA UNIVERSITY \\ UNIVERSITY OF SOUTHERN CALIFORNIA
}

\author{
STANFORD UNIVERSITY \\ UNIVERSITY OF TOKYO \\ UNIVERSITY OF UTAH \\ WASHINGTON STATE UNIVERSITY \\ UNIVERSITY OF WASHINGTON \\ * * * \\ AMERICAN MATHEMATICAL SOCIETY \\ CHEVRON RESEARCH CORPORATION \\ TRW SYSTEMS \\ NAVAL ORDNANCE TEST STATION
}

\footnotetext{
Mathematical papers intended for publication in the Pacific Journal of Mathematics should be typewritten (double spaced). The first paragraph or two must be capable of being used separately as a synopsis of the entire paper. It should not contain references to the bibliography. Manu. scripts may be sent to any one of the four editors. All other communications to the editors should be addressed to the managing editor, Richard Arens at the University of California, Los Angeles, California 90024.

50 reprints per author of each article are furnished free of charge; additional copies may be obtained at cost in multiples of 50 .
}

The Pacific Journal of Mathematics is published monthly. Effective with Volume 16 the price per volume (3 numbers) is $\$ 8.00$; single issues, $\$ 3.00$. Special price for current issues to individual faculty members of supporting institutions and to individual members of the American Mathematical Society: $\$ 4.00$ per volume; single issues $\$ 1.50$. Back numbers are available.

Subscriptions, orders for back numbers, and changes of address should be sent to Pacific Journal of Mathematics, 103 Highland Boulevard, Berkeley 8, California.

Printed at Kokusai Bunken Insatsusha (International Academic Printing Co., Ltd.), No. 6, 2-chome, Fujimi-cho, Chiyoda-ku, Tokyo, Japan.

PUBLISHED BY PACIFIC JOURNAL OF MATHEMATICS, A NON-PROFIT CORPORATION

The Supporting Institutions listed above contribute to the cost of publication of this Journal, but they are not owners or publishers and have no responsibility for its content or policies.

* Paul A. White, Acting Editor until J. Dugundji returns. 


\section{Pacific Journal of Mathematics}

\section{Vol. 16, No. $3 \quad$ BadMonth, 1966}

Gert Einar Torsten Almkvist, Stability of linear differential equations with

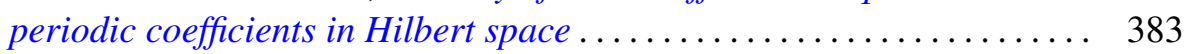

Richard Allen Askey and Stephen Wainger, A transplantation theorem for ultraspherical coefficients ................................ 393

Joseph Barback, Two notes on regressive isols .................. 407

Allen Richard Bernstein and Abraham Robinson, Solution of an invariant subspace problem of K. T. Smith and P. R. Halmos .............. 421

P. R. Halmos, Invariant subspaces of polynomially compact operators . . . . 433

Leon Bernstein, New infinite classes of periodic Jacobi-Perron algorithms.................................... 439

Richard Anthony Brualdi, Permanent of the direct product of matrices .... . 471

W. Wistar (William) Comfort and Kenneth Allen Ross, Pseudocompactness and uniform continuity in topological groups .................. 483

James Michael Gardner Fell, Algebras and fiber bundles . . . . . . . . . . . . 497

Alessandro Figà-Talamanca and Daniel Rider, A theorem of Littlewood and lacunary series for compact groups ..................... 505

David London, Two inequalities in nonnegative symmetric matrices...... 515

Norman Jay Pullman, Infinite products of substochastic matrices ........ 537

James McLean Sloss, Reflection and approximation by interpolation along

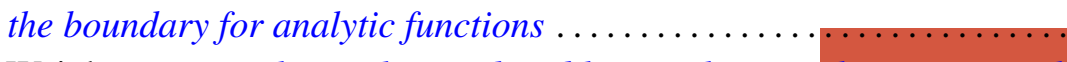

Carl Weinbaum, Visualizing the word problem, with an application to sixth groups................................... 